\title{
Vectorial bending characteristics of long-period grating written in D-shaped fibre
}

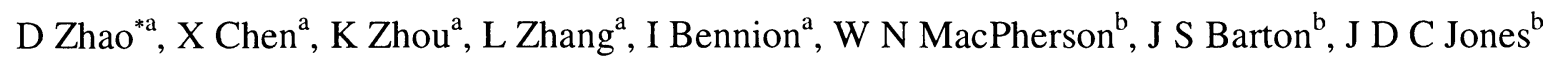 \\ ${ }^{a}$ Photonics Research Group, Electronic Engineering, Aston University, Birmingham B4 7ET, UK; \\ ${ }^{\mathrm{b}}$ School of Engineering and Physical Sciences, Heriot-Watt University, Edinburgh EH14 4AS, UK
}

\begin{abstract}
The curvature- or bend-sensing response of long-period gratings (LPG) UV-inscribed in D-shaped fibre has been investigated experimentally. Strong fibre orientation dependence of the spectral response when such LPGs are subjected to dynamic bending has been observed and is shown to form the basis for new vector sensors.
\end{abstract}

Keywords: Long-period grating, vectorial bending, sensing application

\section{INTRODUCTION}

In recent years, long period fibre gratings (LPFGs), with typical periods of hundreds of micrometres, have attracted much attention for potential use in optical sensing [1,2]. The attractive sensing characteristic of the LPG derives from wavelength shifts or band splitting of its spectral attenuation bands induced by a measurand such as temperature, strain, or bending of the fibre, and is strongly dependent on the structure and composition of the fibre, the LPG period, and the operating wavelength. The LPG offers numerous possibilities for novel sensors for a range of parameters such as refractive index, temperature, strain, and chemical activity $[3,4]$.

Patrick et al. and Liu et al. have proposed and demonstrated accurate bending measurements using LPGs [5, 6]. The experimental results presented in Ref.[5] revealed that the bending response of an LPG written in photosensitive fibre was fibre-orientation-dependent, and the spectral location and strength of the resonance peak changed with fibre orientation, providing an opportunity to realise curvature measurement with direction recognition. Driven by the importance of such measurements in structural integrity monitoring, further investigations have been carried out [7-9]. VanWiggeren et al. demonstrated that the magnitudes and wavelengths of the transmission resonances in curved $\mathrm{CO}_{2}$ laser-induced LPGs are strongly dependent on the axial rotational orientation of the gratings [4, 7]. LPG-based bend sensors written in eccentric core fibre have provided for bipolar curvature determination using a single, easily aligned sensor element [8], and LPGs written in various special fibres, including flat-clad and four-core types, have revealed particularly strong dependence on the direction of the applied bending [9].

Here, we present the results from our study on bend sensing characteristics of LPG structures UV-inscribed in D-shaped fibre (DLPG). The experimental results reveal that the spectral response of DLPG is strongly dependent not just on the degree of the curvature but also on the direction of the applied bending. Moreover, the DLPG exhibits four distinct fibre orientations, associating to the most and the least bend sensitivities. This bend-orientation characteristic is unique in comparison to the above mentioned FBG- and LPG-based bend sensor devices and could be utilised to implement practical bend sensor for real applications.

\section{DLPG}

The D-shaped fibre used in this work was obtained commercially from $\mathrm{KVH}$ : it is elliptical cored, with the flat cladding surface parallel to the major axis of the ellipse and, hence, to the plane of polarization. The curved cladding radius is $125 \mu \mathrm{m}$, and the separation between the centre of the core and the flat cladding surface is $16 \mu \mathrm{m}$.

The fibre was photosensitised by $\mathrm{H} 2$ loading prior to inscription of an LPG structure with a period of $381 \mu \mathrm{m}$ using a $244 \mathrm{~nm}$ UV laser and the point-by-point method. The transmission spectrum of the DLPG was measured using a system incorporating a broadband light source, a polarisation controller and an optical spectrum analyser, with the result shown

* d.zhao@aston.ac.uk; phone 441213593611 ; fax 441213590156 
in Fig.1. In general, two series of peaks in the transmission spectrum due to the fibre birefringence were observed, save for the cases corresponding to light input linearly polarised along either the fast or the slow axis of the fibre. By adjusting the polarisation controller, one series of peaks could be eliminated whilst retaining the other; the extinction ratio of the retained transmission peak at $1629.66 \mathrm{~nm}$ was $8.48 \mathrm{~dB}$ (see the bold curve in Fig 1). Rotating the polarizer through $90^{\circ}$, the first series of transmission peaks was suppressed, and the orthogonally polarised peak at $1645.432 \mathrm{~nm}$ recorded with an extinction ratio of $8.34 \mathrm{~dB}$.

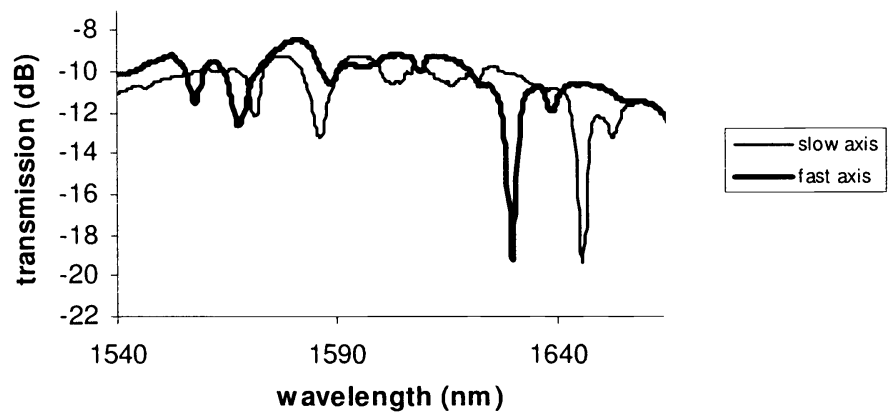

Fig.1 Transmission spectrum of DLPG. Two serials of peaks corresponding to the two polarization states

\section{EXPERIMENTAL RESULTS}

The four-point bending system illustrated in Ref.9 was used to determine the response of the DLPG to bending. Experiments were conducted to determine the spectral responses of DLPGs to variations of curvature for different orientations of the fibre. The grating transmission spectrum during bending was monitored using an optical spectrum analyser with $1 \mathrm{~nm}$ resolution. The fibre was rotated through a series of angles from $0^{\circ}$ to $270^{\circ}$, and the wavelength shift of the bending-induced loss peak was measured at each position.

We observed strong directional bending sensitivity characteristics in the DLPG structure with bending applied at different fibre orientations. For each rotational orientation, the DLPG response was examined for a series of induced curvatures in the range 0 to $8 \mathrm{~m}^{-1}$. To ensure that the axial (x-direction) orientation of the DLPG could be changed without twist, relative to the plane of curvature ( $y-z$ plane), the fibre was marked with a degree scale at both ends. The LPG was axially rotated to a different orientation by first returning the metal plate to zero curvature and then returning the fibre to the same starting position on the metal plate. Fig. 2 shows the six fibre orientations used in the experiments.

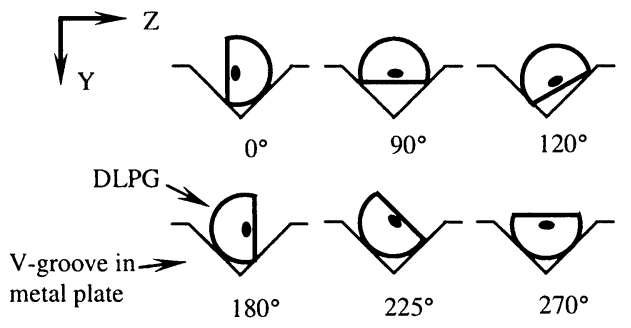

Fig. 2 Different fibre orientations

The DLPG bending responses were found to be strongly dependent on the fibre orientation. With the fibre fixed at $0^{\circ}$ orientation, as shown in Fig. 2, the wavelengths of all of the transmission loss peaks were unaltered by the bending. As the fibre was rotated from $0^{\circ}$ to $120^{\circ}$, the peaks shifted markedly towards shorter wavelengths; with the fibre oriented at $270^{\circ}$, the shifts were towards longer wavelengths.

Fig. 3 (a) and (b) show the wavelength shifts of the two peaks, located initially at wavelengths of $1629.66 \mathrm{~nm}$ and $1645.43 \mathrm{~nm}$, plotted against curvature for six different fibre orientations. It is clear that all of the responses exhibit reasonable linearity, and the peaks show similar shifting trends for a given orientation. The maximum bending sensitivities occurred at $90^{\circ}$ for the negative-going wavelength response, and at $270^{\circ}$ for the positive-going response. For one peak located at $1545.43 \mathrm{~nm}$, wavelength shifts of up to $-20.02 \mathrm{~nm}$ and $+22.78 \mathrm{~nm}$ were observed at an applied curvature of $8.0 \mathrm{~m}^{-1}$ for the $90^{\circ}$ and $270^{\circ}$ fibre orientations, respectively, yielding corresponding bend sensitivities of - 
$2.5 \mathrm{~nm} / \mathrm{m}^{-1}$ and $+2.85 \mathrm{~nm} / \mathrm{m}^{-1}$. At orientations of $0^{\circ}$ and $180^{\circ}$, the maximum wavelength shift detected was less than $1 \mathrm{~nm}$ which is at the limit of the resolution of the optical spectrum analyzer used, and these orientations are, in effect, bendinsensitive. It is important to note that the wavelength shift of the transmission loss peaks is function of the fibre orientation, $i e$ that the bend sensitivity itself is a strong function of orientation, changing from positive through zero to negative values. Moreover, there are four distinct fibre orientations, at which the DLPG exhibits the most and the least bending sensitivities. This bend-orientation feature lends itself to exploitation in the development of vectorial curvature sensing elements for use in smart structural applications, as discussed below.

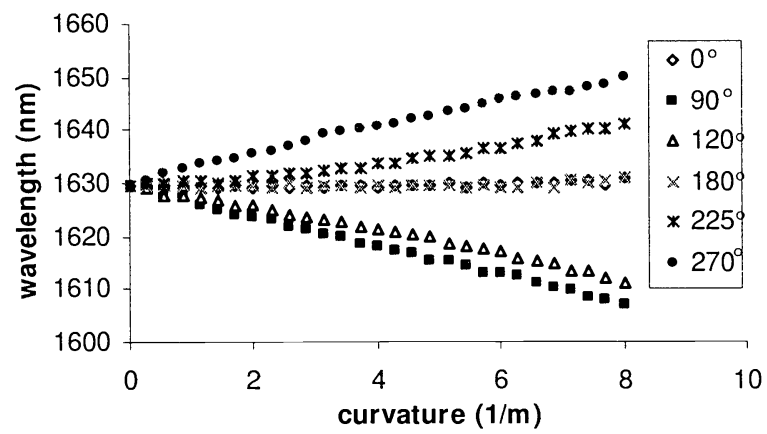

(a)

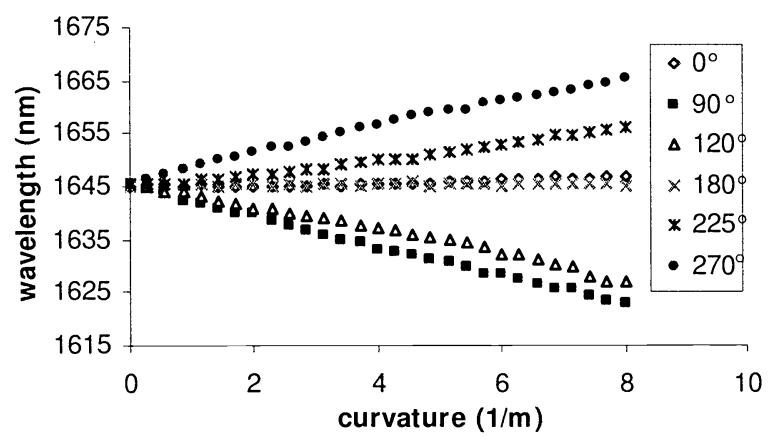

(b)

Fig.3 Wavelength shift of MCF-LPG against curvature at different orientations. (a) peak at 1630nm; (b) peak at $1645 \mathrm{~nm}$

The strong orientational dependence of the bending response of the DLPG derives from the asymmetrical location of the core relative to the geometrical centre of the cladding cross-section. Thus, when the fibre is bent at orientations such as $225^{\circ}$ and $270^{\circ}$, the core is subject to expansive strain so that the LPG peaks move to longer wavelengths. Alternatively, for orientations such as $90^{\circ}$ and $120^{\circ}$, the core undergoes compressive strain, resulting in a shift of the LPG peaks to shorter wavelengths.

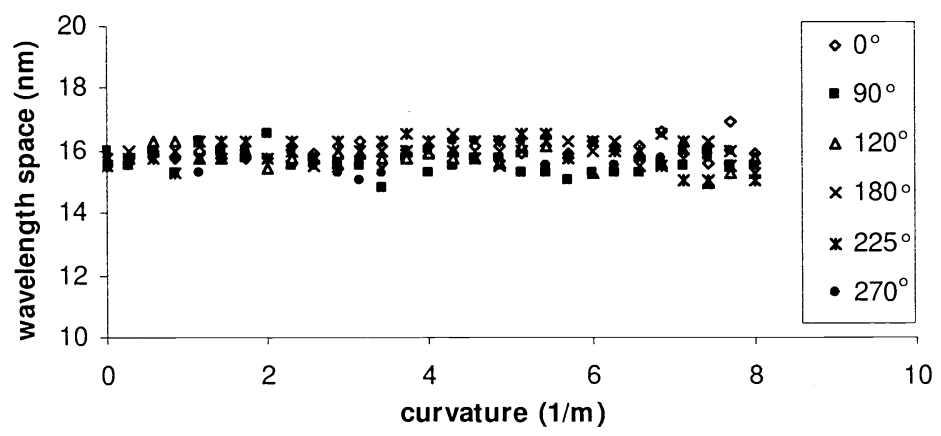

Fig.4 The variation of wavelength space between two peaks under bending

Since the D-shaped fibre is polarisation-maintaining, it is appropriate to determine whether or not the applied bending results in a change of the fibre birefringence. For the DLPG, the wavelength separation between successive orthogonally polarised loss peaks gives a measure of the birefringence. We have measured this separation during the bending experiments with the results shown in fig. 4 for all six fibre orientations. The measured change of the wavelength separation is within $\pm 1 \mathrm{~nm}$, indicating that the bending-induced birefringence change is insignificant. This result is to be expected since in this fibre the origin of the birefringence lies principally in the geometric shape of the core, which is not altered to any significant extent by bending.

\section{POTENTIAL APPLICATIONS}

\subsection{Directional shape sensor}

Recently, there has been increased interest in utilising the bend sensitivity of the LPG for direct shape sensing. In a conventional strain-based shape sensor, curvature is calculated from a linear strain measurement at a known distance from the neutral axis of the structure. In contrast, the LPG exhibits direct bend sensitivity and can be used to measure 
curvature without knowledge of the distance from the neutral axis, rendering it simpler and more versatile in operation. A shape sensor has been reported that employs one LPG optimised for positive, and another for negative, radius of curvature bends $[5,10]$. While this sensor exhibited good sensitivity and repeatability, it required two devices to measure positive and negative curvatures in the same plane, and its response was markedly nonlinear.

These shortcomings may readily be overcome by a single DLPG, embedded at the orientations $\left(90^{\circ}\right.$ and $270^{\circ}$ shown in Fig. 3) corresponding to maximum bending response, which permits the determination of both the magnitude and the direction of induced bending with high resolution and accommodating large dynamic range.

\subsection{Bend-insensitive sensor}

As most LPGs made in conventional fibres are highly sensitive to bend, when they are used for applications sensing other measurands, such as temperature, strain and refractive index, , the problem of cross-sensitivity between the measurand and bend may arise, resulting in inaccurate information. The DLPGs described here may be ideal candidate for these applications. Our results on DLPG bend sensing characteristics clearly show that there are two distinct orientations $\left(0^{\circ}\right.$ and $180^{\circ}$ depicted in Fig. 3), at which the DLPG is totally insensitive to bending. If the DLPG device operated at either these two orientations, it can be used to measure desirable parameter accurately without the effect of bend crosssensitivity. This configuration should be more attractive in comparison with many suggested dual-sensor types, since it offers a direct measurement while the others will require extra grating and measurement procedure for cross-sensitivity decoupling process.

\section{CONCLUSIONS}

In summary, we have investigated the bending sensitivity characteristics of LPG UV-written in elliptical-core D-shaped fibre. The experimental results have clearly indicated that the spectral responses of the DLPG structures depend strongly not just on the curvature amplitude but also the fibre orientation. Four distinct DLPG bend-orientations associated with maximum and minimum bending sensitivities have been identified. The potential applications utilising these unique bend-orientation characteristics have been discussed, showing device advantages over previously proposed fibre grating based sensors.

\section{ACKNOWLEDGEMENT}

This work was supported by the UK Engineering and Physical Sciences Research Council and UK Defence Science and Technology Laboratory.

\section{REFERENCES}

[1] V. Bhatia, and A. M. Vengsarkar, "Optical fibre long-period grating sensors," Opt. Lett., 1996, Vol.21, pp.692-694

[2] V. Bhatia, D. Campbell, R. O. Claus, and A. M. Vengsarkar, "Simultaneous strain and temperature measurement with long-period gratings," Opt. Lett., 1997, Vol.22, pp.648-650

[3] Stephen W. James, and Ralph P. Tatam, “ Optical fibre long-period grating sensors: characteristics and application," Measurement Science and Technology, 2003, Vol.14, pp.R49-R61

[4] Gregory D. VanWiggeren, Thomas K. Gaylord, Donald D. Davis, Mohammad I. Braiwish, Elias N. Glytsis, and Emmanuel Anemogiannis, "Tuning, attenuating, and switching by controlled flexure of long-period fiber gratings," Optics Lett., 2001, Vol.26, No.2, pp.61-63

[5] H. J. Patrick, C. C. Chang, and S. T. Vohra, “Long period fiber gratings for structural bend sensing," Electron. Lett., 1998, Vol.34, No.18, pp. 1773-1774

[6] Y. Liu, L. Zhang, J. A. R. Williams, and I. Bennion, " Optical bend sensor based on measurement of resonance mode splitting of long-period fiber grating”, IEEE Photon. Tech. Lett., 2000, Vol.12, No.5, pp. 531-533

[7] G. D. VanWiggeren, T. K. Gaylord, D. D. Davis, E. Anemogiannis, B. D. Garrett, M. I. Braiwish, and E. N. Glytsis, "Axial rotation dependence of resonances in curved CO2-laser-induced long-period fibre gratings," Electron. Lett., 2000, Vol.36, No.16, pp.1354-1355

[8] H. J. Patrick, "Self-aligning, bipolar bend transducer based on long period grating written in eccentric core fibre," Electron. Lett., 2000, Vol.36, No.21, pp.1763-1764

[9] D. Zhao, L. Zhang, and Ian Bennion, "Implementation of vector bending sensors using long-period gratings UV-inscribed in flatclad and 4-core fibres," Conf. Proc. Optical Fibre Sensors-16 (OFS-63), 2003, pp.794-797

[10] H. J. Patrick, and S. T. Vohra, "Directional shape sensing using bend sensitivity of long period fibre gratings," Conf. Proc. Optical Fibre Sensors-13 (OFS-13), 1999, pp.561-564 